Conclusions Rates of extubation failure were not significantly different between the groups. HFNC resulted in significantly less nasal trauma than NCPAP. This benefit may need to be considered in post-extubation respiratory support for preterm infants.

\section{MASK OR NASAL TUBE TO PROVIDE POSITIVE PRESSURE VENTILATION (PPV) TO PRETERM INFANTS IN THE DELIVERY ROOM (DR)-THE MONT TRIAL}

doi:10.1136/archdischild-2012-302724.0138

${ }^{1} \mathrm{COF}$ Kamlin, ${ }^{2} \mathrm{~K}$ Schilleman, ${ }^{1} \mathrm{JA}$ Dawson, ${ }^{2} \mathrm{E}$ Lopriore, ${ }^{1} \mathrm{~S}$ Donath, ${ }^{1} \mathrm{GM}$ Schmoelzer, ${ }^{2} \mathrm{FJ}$ Walther, ${ }^{1} \mathrm{PG}$ Davis, ${ }^{2} \mathrm{AB}$ te Pas. ${ }^{1}$ Royal Women's Hospital, Melbourne, VIC, Australia; ${ }^{2}$ Leiden University Medical Center, Leiden, The Netherlands

Background For initial PPV a face mask is used, but obstruction and leak often occurs. A nasal tube is an alternative interface in the DR to provide PPV. Its safety and efficacy have not been tested in a large RCT in extremely preterm infants.

Objective To determine whether a single nasal tube is more effective than face mask during the stabilisation of infants born between 24 and 29 weeks.

Design and methods An RCT performed in Melbourne and Leiden. Infants were randomized immediately prior to birth to receive PPV using a T-piece with either a nasal tube or round mask. Resuscitation guidelines were standardised. Criteria for intubation: cardiac compressions, apnea, CPAP $>7 \mathrm{cmH}_{2} \mathrm{O}$ and $\mathrm{FiO}_{2}>0.4$.

Primary outcome: intubation in the first 24 hours from birth. Results In total 368 infants were randomized. Baseline variables, primary and secondary outcomes were similar (table).

Abstract 138 Table 1 Baseline characteristics and outcome

\begin{tabular}{llll}
\hline & Mask (N= 187) & Nasal Tube (N= 181) & OR (95\% Cl) \\
\hline GA (wks) & $27(26-29)$ & $27(26-28)$ & - \\
Birthweight (g) & $1000(807-1196)$ & $946(780-1182)$ & - \\
Intubation in first 24hrs & $102(54)$ & $97(54)$ & $0.96(0.64-1.45)$ \\
Intubation in DR & $58(31)$ & $41(23)$ & $0.65(0.41-1.04)$ \\
Air Leak & $14(8)$ & $11(6)$ & $0.80(0.35-1.81)$ \\
Total (MV+CPAP) days of & $13(6-41)$ & $16(4-41)$ & NS \\
ventilation & & & $0.96(0.62-1.47)$ \\
Death or BPD & $68(36)$ & $64(35)$ &
\end{tabular}

Conclusions In infants $<30$ weeks gestation receiving PPV in the $\mathrm{DR}$, there were no differences in short term outcomes using the nasal tube compared to the face mask.

\section{RESEARCH NETWORKS IN PEDIATRIC PRIMARY CARE:} EAPRASNET

doi:10.1136/archdischild-2012-302724.0139

'S del Torso, ${ }^{2} \mathrm{D}$ van Esso, ${ }^{3} \mathrm{~A}$ Hadijpanayis, ${ }^{4} \mathrm{Z}$ Grossman. ${ }^{1}$ ULSS 16, Padova, Italy; 2Primary Care Center 'Pare Claret', Barcelona, Spain; ${ }^{3 P e d i a t r i c ~ D e p a r t m e n t, ~ L a r n a c a ~}$ General Hospital, Larnaca, Cyprus; 'Maccabi Health Services, Tel Aviv, Israel

EAPRASnet is the European Academy of Paediatrics Research in Ambulatory Setting Network, whose members are European primary care paediatricians committed to research in their practices that expressed their will to join a research network.

The network is the research laboratory of the primary care setting and in the latest study, EAPRASnet has enrolled a total of 685 pediatricians from 21 countries.

A recruitment survey showed that areas of most interest for research were: quality of care indicators, communication with parents, obesity, attention deficit hyperactivity disorder and effective well child care. Main incentives for participation in a research project were interest in the topic (81\%) and effort to improve quality of care $(71 \%)$. Lack of time was the leading reported obstacle $(72 \%)$.

A second electronic survey assessing primary care pediatricians' estimations and practices regarding parents' vaccination refusal was sent to 395 EAPRASnet members, with a response rate of $87 \%$. Of respondents who vaccinate in their clinic, 93\% estimated the total vaccine refusal rate less than $1 \%$. Sixty nine percent of all respondents prefer a shared decision making approach to handle parents' vaccine hesitancy.

A recent survey on Use of Antibiotics in Upper Respiratory tract infections has been performed to evaluate European primary care paediatricians' knowledge, attitudes and practice regarding antibiotic prescribing for upper respiratory infections in order also to identify targets for future intervention studies.

\section{RESEARCH NETWORKS IN PEDIATRIC PRIMARY CARE: TRANSITION TO REALITY}

doi:10.1136/archdischild-2012-302724.0140

T DeWitt. Pediatrics, Cincinnati Children's Hospital Medical Center, University of Cincinnati College of Medicine, Cincinnati, OH, USA

Research networks in pediatric primary care, often called practicebased research networks (PBRN) in the United States, have an increasingly important role in the spectrum of research that spans from basic research to practical and effective application in primary care settings. In addition to assessing efficacy, PBRN's are particularly critical in assessing effectiveness in the real world of community practice - the final step in translating new discovery into practice. Key elements of effective translation that can be assessed by PBRN's include rapidity of adoption, adherence, and quality improvement systems to assure consistent application. This presentation will discuss PBRN's in the context of the National Institute of Health's "Roadmap for Medical Research" and an emphasis on translational research. Examples of the use of pediatric PBRN's in this capacity, including ADHD and asthma, with a focus on effectiveness will be discussed.

\section{TRANSCUTANEOUS ELECTRICAL STIMULATION (TES) AND INTRACTABLE CHRONIC CONSTIPATION IN CHILDHOOD}

doi:10.1136/archdischild-2012-302724.0141

${ }^{1,2,3}$ Y Y Yik, ${ }^{3,4} \mathrm{JM}$ Hutson, ${ }^{3} \mathrm{BR}$ Southwell. 'Department of General Surgery, Faculty of Medicine, University of Malaya, Kuala Lumpur, Malaysia; '2Department of Paediatrics, University of Melbourne; ${ }^{3} G u t$ Motility and Research Laboratory, Murdoch Children's Research Institute; ${ }^{4}$ Department of Urology, Royal Childrens Hospital Melbourne, Melbourne, VIC, Australia

Background and aims Intractable chronic constipation in the form of slow-transit constipation (STC) often resistant to medical treatment. Some children required appendicostomy for antegrade enemas to improve symptoms. Transcutaneous electrical stimulation (TES) was used by physiotherapists to overcome STC in children successfully. This study aimed to examine the effectiveness of home-based TES when STC children were trained by a naïve clinician. We hypothesized TES would improve symptoms with reduce laxative use.

Methods A prospective study (2009-2011) whereby a surgeon was trained to deliver TES method to STC children/parents, who then self-administered TES at home ( $1 \mathrm{hr}$ day $\times$ 6 months) using a battery-powered interferential stimulator. Daily continence diary (including laxative use) was recorded before and throughout TES; PedsOL4.0 questionnaires and gastrointestinal nuclear transit scintigraphy (NTS) were completed before and after TES. Appendicostomy for antegrade enemas was offered if TES failed to improve 